Témoigner Témoigner. Entre histoire et mémoire

Getuigen Revue pluridisciplinaire de la Fondation Auschwitz

$123 \mid 2016$

Traduire le témoignage

\title{
Mémorial de la guerre patriotique de Vukovar
}

The Memorial Centre of Homeland War Vukovar, Croatia

\section{Iva Šušić Degmečić}

Traducteur : Carine Chauran

\section{(2) OpenEdition}

\section{Journals}

Édition électronique

URL : https://journals.openedition.org/temoigner/5472

DOI : $10.4000 /$ temoigner.5472

ISSN : 2506-6390

Éditeur :

Éditions du Centre d'études et de documentation Mémoire d'Auschwitz, Éditions Kimé

Édition imprimée

Date de publication : 1 octobre 2016

Pagination : 193-196

ISBN : 987 2-9600926-4-6

ISSN : 2031-4183

Référence électronique

Iva Šušić Degmečić, « Mémorial de la guerre patriotique de Vukovar», Témoigner. Entre histoire et

mémoire [En ligne], 123 | 2016, mis en ligne le 02 novembre 2021, consulté le 04 novembre 2021. URL: http://journals.openedition.org/temoigner/5472 ; DOI : https://doi.org/10.4000/temoigner.5472

Tous droits réservés 


\section{Site mémoriel}

\section{MÉMORIAL DE LA GUERRE PATRIOTIQUE DE VUKOVAR}

Reconstruction des camps de concentration Stajicevo

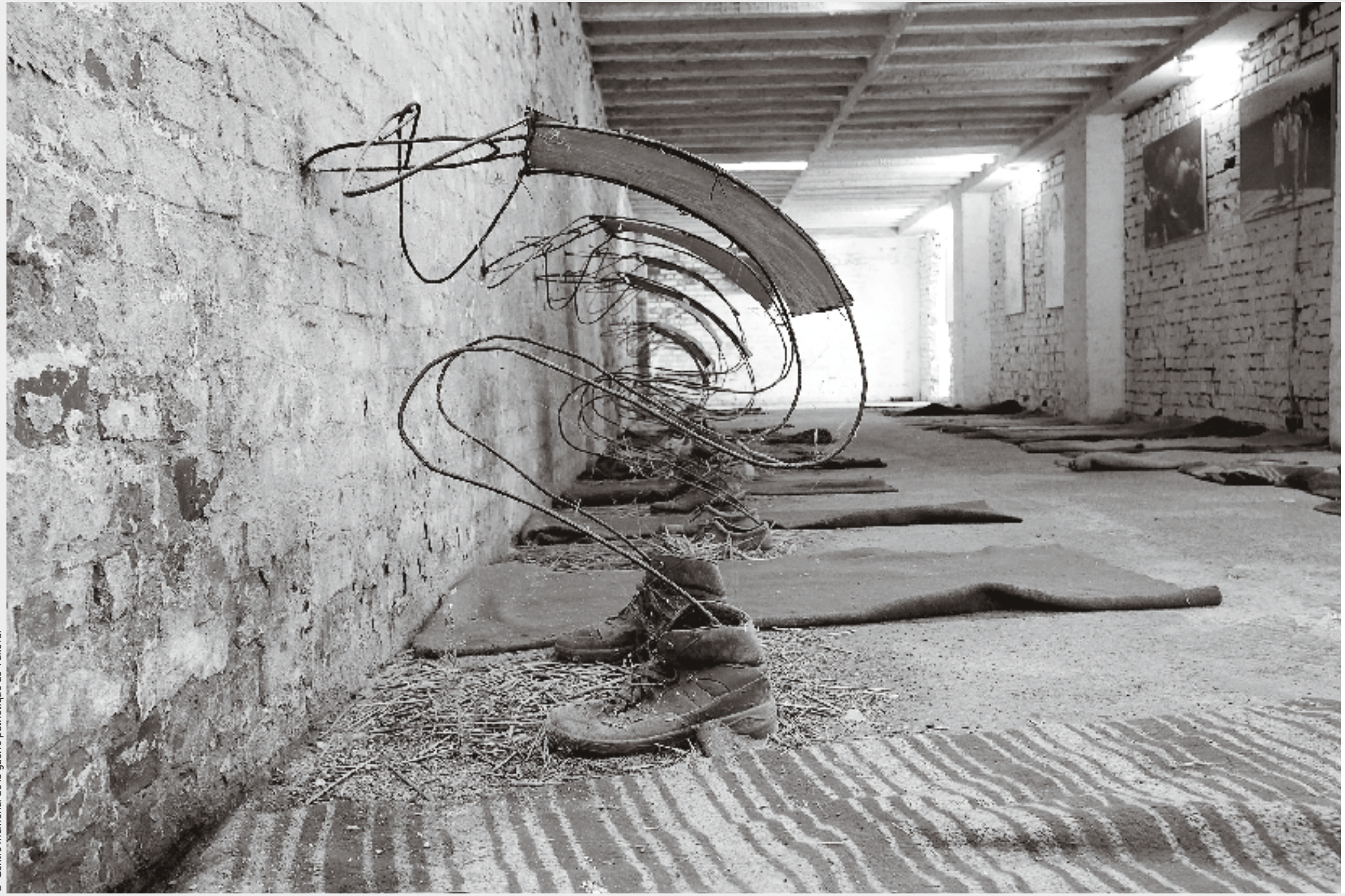

Le siège militaire de Vukovar s'est déroulé du 25 août au 20 novembre 1991. Durant du ces tris a encerclée et toutes les voies daccès étaient der a la ville que a la ville ét laquelle les assiégés étaient ravitaillés, et qui villages de Bogd, en traversant les villages de Bogdanovci et Marinci.

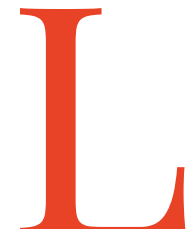

e $1^{\text {er }}$ octobre, l'Armée populaire yougoslave (JNA) prenait le voie de communication restante avec Vukovar (Nazor, 2011, p. 219). Malgré fait que la ville étaitalors totaleme erné il fallute était alors totalement la JNA pourvenirà à unot denià des Croates (Nazor, 2011, p. 219).

Le siège organisé par la Serbie, la JNA, la défense territoriale de Serbie et les unités paramilitaires serbes a mené a destruction complète de Vukovar. Le nónés hernes de la Garde nationale our, membres chus de toutes les récions de la Crotie (Nazor, 2011, p. 220). D’après un croatie (Nazor, 201,'p.22́s. D'apres une source de la 37613 tArmee disposait par contre de 37 cis troupes en Slavonie orientale, port de troupes, 418 untés d'ansport de troupes, 418 unités d'artillerie et 380 unités d'artille et 380 unités d'artillerie antiaérienne quilibre des forces en termes d'armes et d'équipement militaire a contribué à la perception de Vukovar come ànole d'indépendance croate- appelée dindénendance croate - appelée (Homeland Warn (guerre patriotique) des «héros nationaur.

Même si la plupart des unités de défenseurs organisées par le biais de défensenrs organisees par le biais de d'auna véritable for hisposiont taire, elles sont parvention milltaire, elles sont parvenues à assurer la défe de la ville pendant trois mois. La JNA et les Serbes ont finalement pris le contrôle de Vukovar, non sans plan militaire que politiqu tant sur le tiqun millté que politique. Les statistela dera Res ont perdu plusieurs milliers de Serbs ont perdu plusieurs milliers de dés (Marijan, 2002 p. 398). Le fait que dés (Marijan, 2002, p. 398). Le fait que rain, tel que l'avait prévu initialent rain, tel que lavait prevu initialement le Hé con rative socia s. effet dévastateur surlemares vation des soldats Quiplusest, pendant la périod del'invasion, est, pendant 
(1) «Story about a town », de
Sinisisa Glavaševic, journaliste Sinisa Glavaseveric, jour
de la radio croate de
Vulovarar capturé dans Vukovar, capturé dans
l'hopptial de Vulovar et tué à Ovcara après l'occupation durant le siège et la Nazor 2011, p. 402).
•• été mobilisés dans d'autres régions de p. Croatie également (Jurcević 1993 p. 496), ce qui a été crucial pour la deVukovar a ale du pays. La tragede dans l'attitude de la communautéinterderion nationale, qui aconno République.

es événements suivant l’occupation de Vukovar - massacres de pation de Vukovar - massacres de sexuelles, détentions tortures, violences tration, détention tor tration, nettoyage ethnique et pillages ont forgé l'identité de la ville Siniš ont forgé licentité de la ville. Siniša de Vukovar, a écrit :

Quelqu'un a simplement tout volé [...]. Votrepasséest tout bonnement détru et il ne vous reste plus rien. Vous deve tout reconstruire, à commencer par racines, et en continuant avec vos présent. Et s'il vous reste des forces, preus devez ses investir dans le futur.

L'objectif du Centre mémorial de a guerre patriotique de Vukovar s'inscrit bien dans lesprit de Glavasevic : esquisser le futur de la ville en relian son passé et son présent. Creè en 2013 civils et à la ville elle-mên sold ts, aux civils et à la ville ello-mène, le Centre à la racre àla mén à a a lededucation, Parmi ses nombreuses antivés, lon Parni ses nombreuses activités, lon retient lorganisation de cérémonies collection d'artefacts et de docires, historiques, la préparation d'expostions enes, la preparation d'exposi-

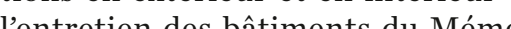

rial. Le Centre présente également une xposition permanente, «Mémoire de la guerreparioticue la ba gur à partir de mai 1991 et la rébellion des a partiŕ nes insurgés serbes à Borovo Selo, les pre-

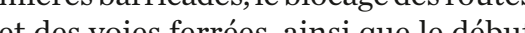
de la défense organisée de Vukovar. Les cirteur peuventenoutrevoir lexposipendant la guere, la reconstruction pendant la guerre, la reconstruction Stajićevp de cham champ de bataille "Brigade renforcée reur. Les camps ont été reconstitú dans la cave d'une bara reconstitús pour illustre dune baraque militaire phe photos, les conditions de vie a l'époque Le Centre con liés à la Guerre d’indép dos colloques ếs à la Gurre dindépend

Alors que le Mémorial estencore très récent, son implication dans l'éducation Ministe des Sciete reconnue par le tes Sports croate, de leducation tes Sports croate, qui a ainsi rendu Vukovary pour tous létè des déch de croas primaires de la République de Croatie. Le programme inclut la importants pour la contes némoraux mémoire cou

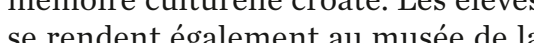
ville de Vukovar ̀̀ Chât nusée de ruou musé a chó qu'au musée archéchlogique de Vučedo La visite dure deux jours et se termine La visite dure deux jours et se termine par un cours déducation a la paix qui connaise las éla

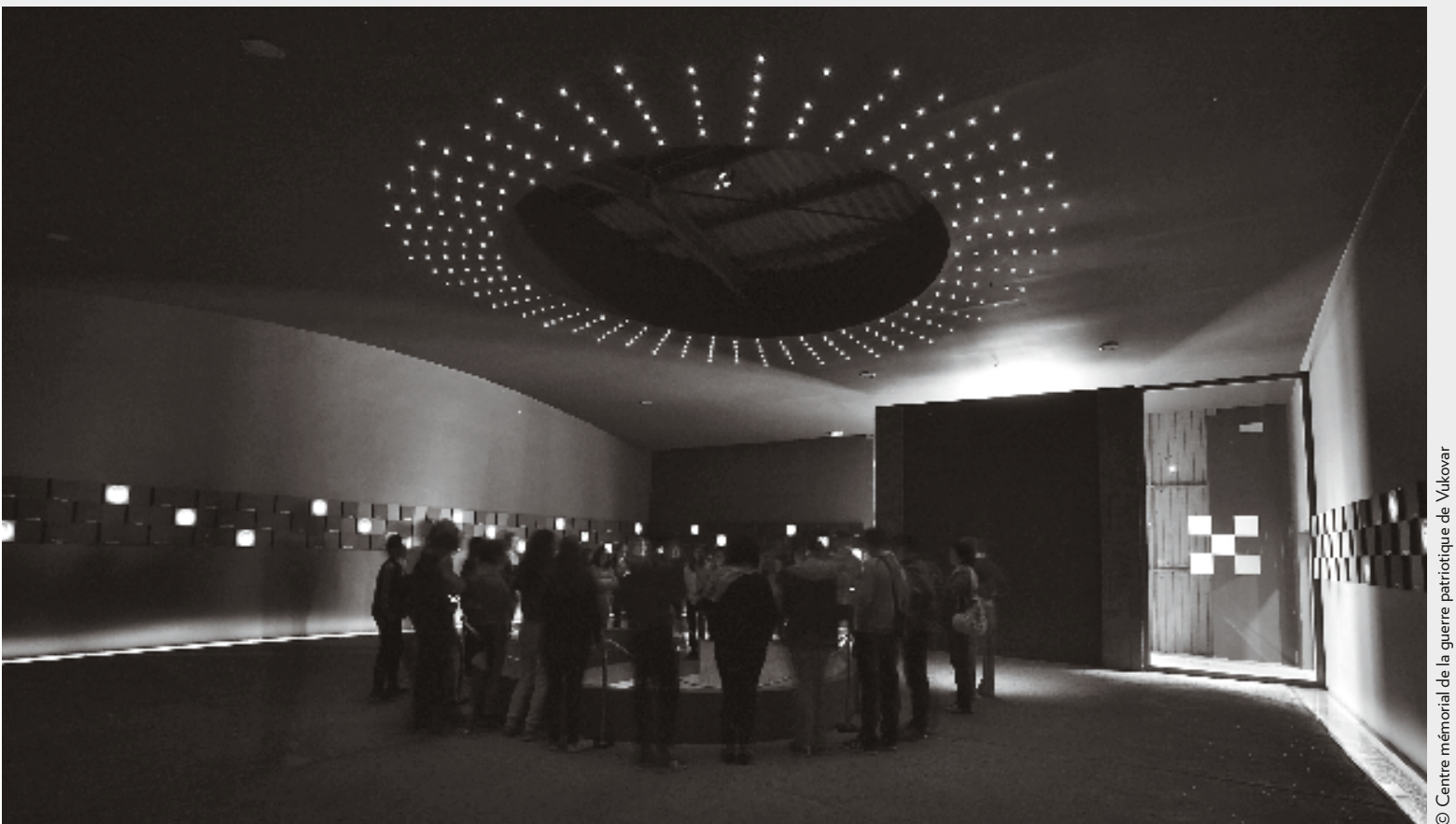

Trente-cinq ans se sont écoulés et, Vukovar, « les traces de la guerre son . 128) La supicion. (Ź, toujours présen tes da le ceen resprit eville profondén

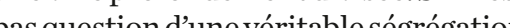
pas quition phique, lon constate bien la division sociale dans les magasins, les bars, les estaurants, les naternelles, les écoles prost les evenenents sportifs. Ce prour e pour sour ecón tilisent des manuels diffés sents un concentrontsure illes ńles à Vukovar ont mêne des entrées
Depuis 2003, plusieurs initiatives ont té lancées pour fonder une «Nouvelle école ouverte a des en cros. cis pas été volonté politique. Il sem de labsence de cessus de réintégration pacifique lancé en 1998, n' pas tran só Vue, lance encé ences poursuite des ciminels, enstits les conditions críáninels, constituent table pix, à préalables à une veria la péconc a aniation ala reconciliation. Le Centre mémorial etde commémoration auxjerceśn rations.

Iva Šušíc Degmečic mémorial de la guerre patriotique de Vukovar
Traduit de langlais par Carine Chauran $\uparrow$ Centre de Vukovar. 


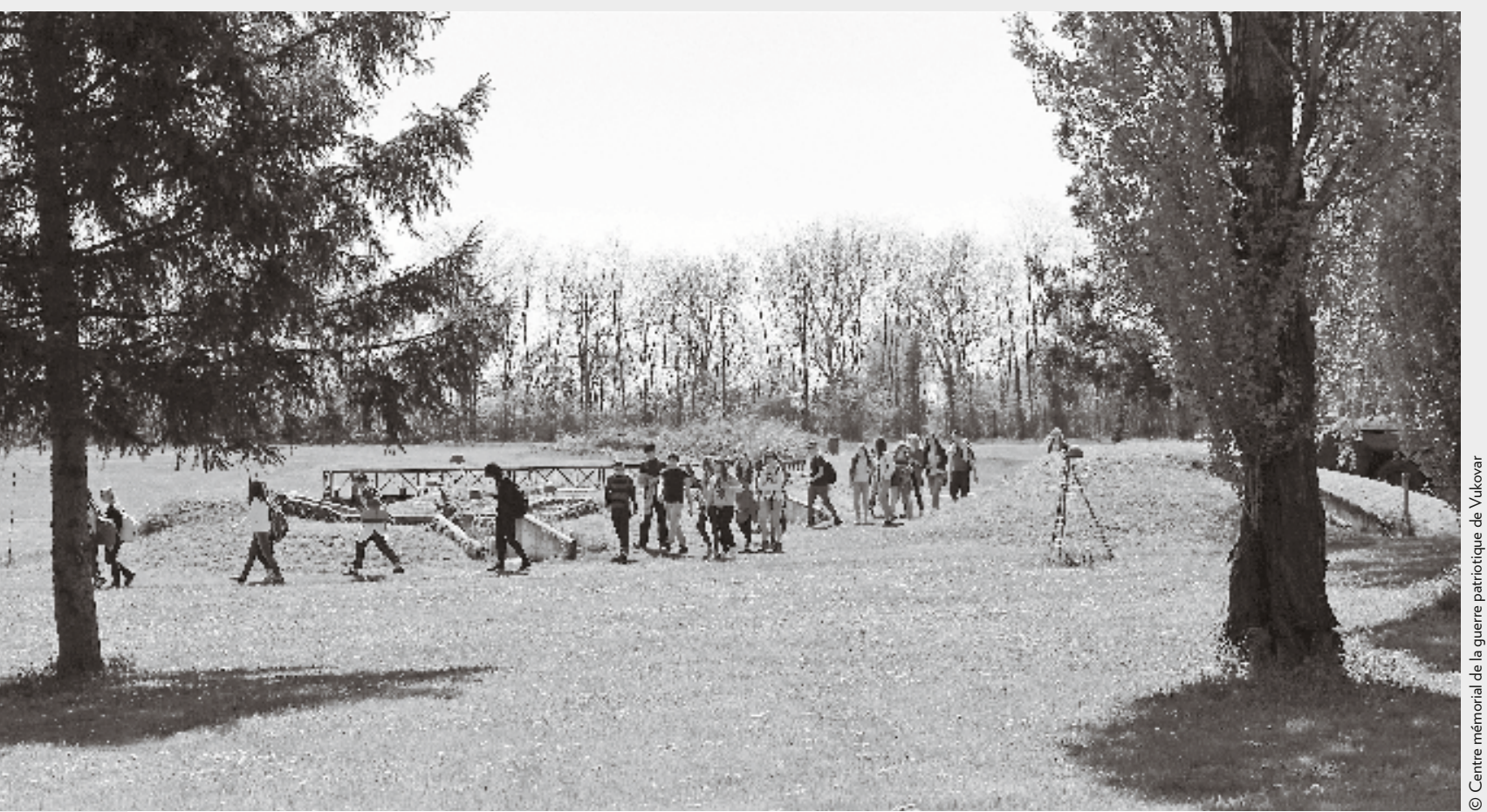

Reconstitution du champ de bataille « Brigade renforcée en défense ».

\section{Bibliographie}

$\diamond$ Jurčević, Josip (1993) : «Vukovarski otpor srpskoj ratnoj agresiji na Hrvatsku 1991... » [La résistance de Vukovar à l'agression serbe contre la Croatie en 1991], Društvena istraživanja, vol. 2, n 2-3, p. 479-499.

$\diamond$ Marijan, Davor (2002) : «Bitka za Vukovar 1991 » [La Bataille de Vukovar 1991], Scrinia Slavonica, n² 2, p. 367-401.

$\diamond$ Marijan, Davor (2012): «Zamisao i propast napadne operacije Jugoslavenske narodne armije na Hrvatsku u rujnu 1991. godine " [Lidée et l'échec de l'opération d'assaut de l'Armée populaire yougoslave contre la Croatie en septembre 1991], Časopis za suvremenu povijest, vol. 44, $n^{\circ}$ 2, p. 251-275.

$\diamond$ Nazor, Ante ; Marijan, Davor ; Tuković, Ana Holjevac ; Živić, Dražen ; Grujić, Ivan (2016) : Bitka za Vukovar (uzroci i posljedice) [La Bataille de Vukovar (causes et conséquences)], Zagreb, Centre croate de commémoration et de documentation sur la guerre patriotique.

$\rightarrow$ Nazor, Ante (2011): Greater-Serbian Aggression against Croatia in the 1990s, Zagreb, Centre croate de commémoration et de documentation sur la guerre patriotique.

$\diamond$ Sučić, Stjepan (2011) : «Značaj obrane Vukovara u stvaranju hrvatske države » [Limportance de la défense de Vukovar pour la création de l'état croate], National security and the future, vol. 3, $n^{\circ} 12$, p. 1-59.

$\diamond$ Živić, Dražen (2008): "Consequences of the Serbian Aggression as a Determinant of Vukovar's Postwar Reconstruction and Development », Društvena istraživanja : časopis za opća društvena pitanja, vol. 17, n 1-2, p. 27-50. 